\title{
Evolution of content - the Concise Communication
}

A central goal of the Editorial group at Johns Hopkins is to accelerate the transmission of cutting-edge biomedical science. There is often a tension between the timeliness of publication and the completeness of a story. We are interested in stories that are complete, and even if brief, a part of the message should be definitive. The definition of definitive is often in the eye of the beholder (i.e., editors and reviewers) and the mandate of the publication venue. We will maintain a rigorous standard for publication in the JCI but do wish to solicit brief, elegant, and captivating reports - memorable scientific short stories.

In this spirit, we have reconfigured the Brief Report category and renamed it Concise Communication, with the goal of publishing brief but complete pieces of science. The Concise Communication has a 4,000-word limit, with up to four display items (combinations of figures and tables) and may include a supplement. The final format of the Concise Communication is: Abstract, Introduction, combined Results and Discussion, followed by Methods. The Methods section may be entirely online, but for clinical studies, the study approval information must be reported in the main text. The supplements for Concise Communications will not have a length or word limit. The change from the Brief Report to Concise Communication format will occur with the November 2017 edition of the JCI, therefore, any submitted Brief Reports published after this date will appear in the Concise Communication format.

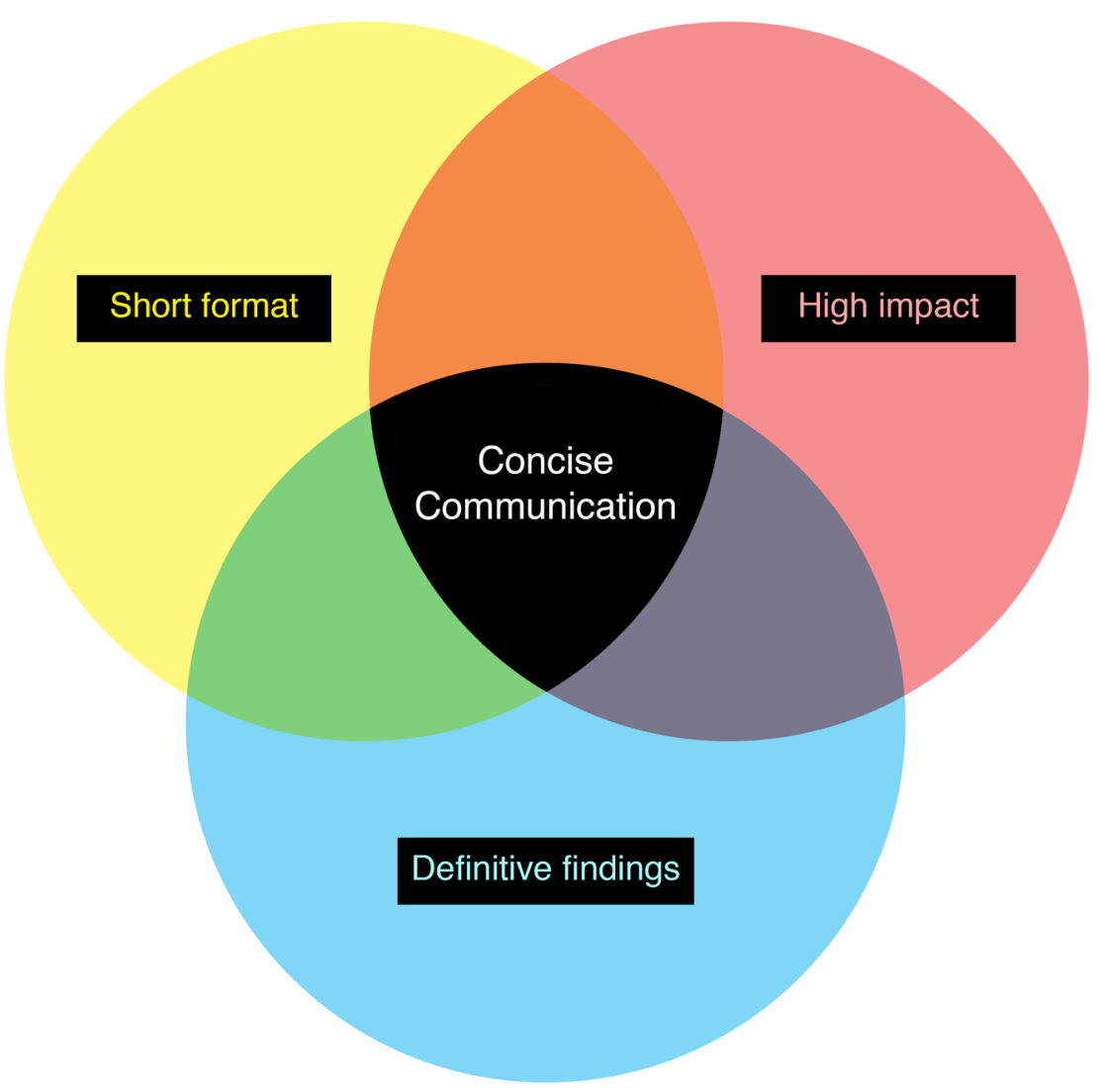

Another goal of our Board is to make submission to the JCI easier for authors. In order to streamline the submission process, we are allowing for more flexibility in the format of original science that comes to the JCI. We know that our authors have a number of venue choices for publishing their work, and liberalizing the format will allow authors to avoid having to change their manuscript for submission to the JCI. Revisions to align with the JCI's format will only be required of manuscripts that are considered for publication. We seek the timely publication of discoveries in basic and clinical biomedical science that will advance the practice of medicine. We look forward to the opportunity to evaluate your work.

\section{Gordon F. Tomaselli Editor}

